\title{
Influence of material properties on the performance of blast-loaded steel plates with pre-cut defects
}

\author{
Benjamin S. Elveli ${ }^{1,2 *}$ Tore Børvik ${ }^{1,2}$, and Vegard Aune ${ }^{1,2}$ \\ ${ }^{1}$ Structural Impact Laboratory (SIMLab), Department of Structural Engineering, NTNU - Norwegian \\ University of Science and Technology, Norway \\ ${ }^{2}$ Centre for Advanced Structural Analysis (CASA), NTNU, Norway
}

\begin{abstract}
Experimental and numerical investigations are carried out to determine how thin steel plates with pre-cut defects behave under blast loading. The defects considered in this study are represented by four square holes, symmetrically distributed around the centre of the target plates. The target plates were manufactured from two types of steel, i.e., a dual-phase medium strength steel and a high-strength martensitic steel. A shock tube facility was used to expose the plates to blast-like loading conditions. The experiments showed that both the blast resistance and the corresponding fracture mode changed with material properties. Numerical simulations were performed using the finite element code LS-DYNA, where the numerical results were found to be in good agreement with the experimental data in predicting the ductile fracture during the blast-structure interaction. The numerical simulations confirmed that significant work hardening will distribute the plasticity throughout the plate material during deformation, while limited work hardening will tend to localize the plasticity that results in earlier fracture.
\end{abstract}

\section{Introduction}

Research on blast-resistant design has historically mostly been focusing on military installations with thick-walled massive constructions [1]. Civilian structures are often more lightweight and flexible than traditional fortified structures. As a result, it is seen an increased interest in studies on thin-walled structures [2,3]. These structures may also contain geometrical defects (e.g. for design purposes or by fragments accelerated by a blast wave that impacts the target plate prior to the blast pressure). This results in local areas of high stress concentrations and a more complex behaviour under blast loading.

Moreover, from a protective point of view, a common perception is that increased strength leads to increased protection against external loading. However, this is not always the case. A high strength generally comes at the price of a reduction in ductility. This strength-ductility trade-off has been a long-standing dilemma in materials science [4] and should be considered

\footnotetext{
*Corresponding author: benjamin.s.elveli@ntnu.no
} 
in protective design. This motivates detailed studies on the influence of material properties on the blast performance of thin steel plates with pre-cut defects.

\section{Materials}

In this study, two different steel materials were investigated. Docol 600DL is a dual-phase, medium-strength steel that undergoes significant work hardening and plastic deformation before fracture, while Docol 1400M is a pure martensitic high-strength steel characterized by higher strength, less work hardening and lower ductility. Both materials are cold-rolled and produced by the Swedish manufacturer SSAB [5].

Dual-phase steels consist of a soft ferrite matrix with islands of hard martensite. This unique combination yields a material with a high ductility due to the ferrite, and a strength depending on the amount of martensite. Hence, an increased amount of martensite will lead to an increase in strength, but also to a decrease in ductility [6]. The chemical composition for both materials is given in Table 1.

Table 1. Chemical composition of Docol 600DL and Docol 1400M (in wt.\%) [5].

\begin{tabular}{|c|c|c|c|c|c|c|c|}
\hline Material & $\begin{array}{c}\mathrm{C} \\
(\max \%)\end{array}$ & $\begin{array}{c}\mathrm{Si} \\
(\max \%)\end{array}$ & $\begin{array}{c}\mathrm{Mn} \\
(\max \%)\end{array}$ & $\begin{array}{c}\mathrm{P} \\
(\max \%)\end{array}$ & $\begin{array}{c}\mathrm{S} \\
(\max \%)\end{array}$ & $\begin{array}{c}\mathrm{Al} \\
(\min \%)\end{array}$ & $\begin{array}{c}\mathrm{Nb}+\mathrm{Ti} \\
(\max \%)\end{array}$ \\
\hline Docol 600DL & 0.1 & 0.4 & 1.50 & 0.01 & 0.002 & 0.04 & - \\
\hline Docol 1400M & 0.2 & 0.4 & 1.60 & 0.02 & 0.01 & 0.015 & 0.1 \\
\hline
\end{tabular}

Quasi-static tension tests of the Docol 600DL material was conducted by Aune et al. [7]. Dog-bone test specimens were manufactured in $0^{\circ}, 45^{\circ}$ and $90^{\circ}$ relative to the rolling direction of the target plates, and a slightly anisotropic behaviour was found both in flow stress and elongation to fracture For the Docol 1400M material, a new set of quasi-static tension tests was performed in Ref. [8], using the same test setup as in Ref. [7]. The corresponding engineering stress-strain curves to fracture are given in Fig. 1. Here, the engineering fracture strain for the Docol $1400 \mathrm{M}$ material varied between 0.025 and 0.045 , and for the Docol 600DL material between 0.22 and 0.28 . Since an isotropic behaviour was assumed for both materials, the tension tests in the $0^{\circ}$ direction were used for calibration of the constitutive model. Due to the large variations within the tension tests in the $0^{\circ}$ direction for Docol $1400 \mathrm{M}$, it was decided to calibrate material parameters for both the tension test giving the largest and the smallest engineering strain at failure within the $0^{\circ}$ direction (see Fig. $1 \mathrm{a}$ and Table 2).

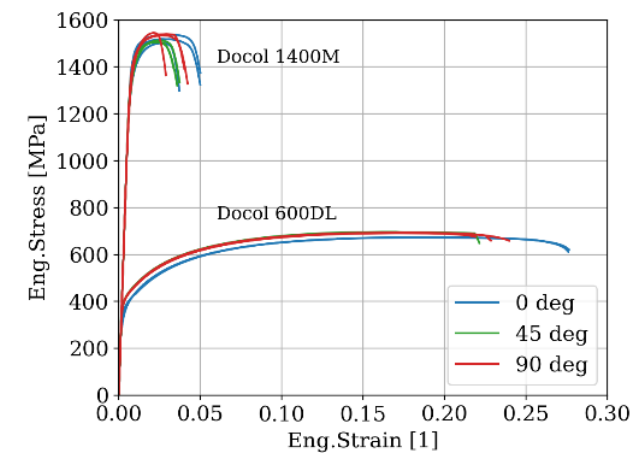

(a)

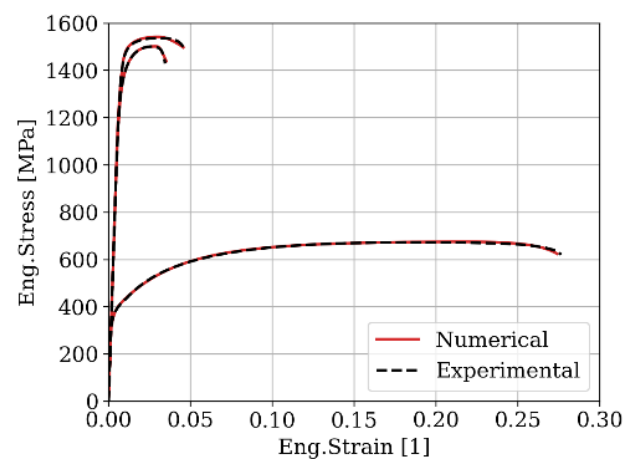

(b)

Fig. 1. Engineering stress-strain curves for Docol 600DL and Docol 1400M: (a) experimental curves and (b) numerical results applying the fitted material parameters in the simulations. 


\section{Experimental setup}

All blast tests were carried out in the SIMLab Shock Tube Facility (SSTF) at NTNU. The performance of the SSTF in generating a blast-like environment is thoroughly described in Ref. [9]. The overall dimensions and features of the SSTF are given in Fig. 2a. The dynamic response of the target plates is recorded during the entire blast-loading event using two highspeed cameras (Phantom v2511) with a sampling rate of $37 \mathrm{kHz}$. Additionally, the pressure is recorded at the two pressure sensors indicated in Fig. 2a, both with a sampling rate synchronized with the high-speed camera recordings and at $500 \mathrm{kHz}$. The synchronization allows for a time axis related to the loading history of the target plates.

All target plates used in this study had a nominal thickness of $0.8 \mathrm{~mm}$ and a blast-exposed area of $300 \mathrm{~mm} \times 300 \mathrm{~mm}$, corresponding to the inner cross section of the SSTF. Four preformed $60 \mathrm{~mm} \times 60 \mathrm{~mm}$ square holes were cut out of the target plates, as illustrated in Fig. 2b. Plates of both materials were exposed to blast intensities resulting from firing pressures of 15 bar, 25 bar and 35 bar. These three firing pressures correspond to a peak reflected pressure on the surface of the target plates of approximately $0.62 \mathrm{MPa}, 0.83 \mathrm{MPa}$, and 1.19 $\mathrm{MPa}$, followed by an exponential decay over a period of 45-75 ms.

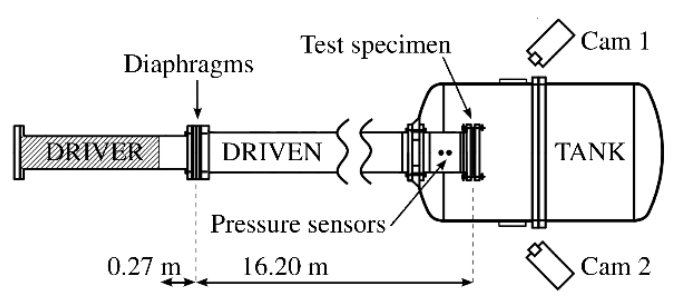

(a)

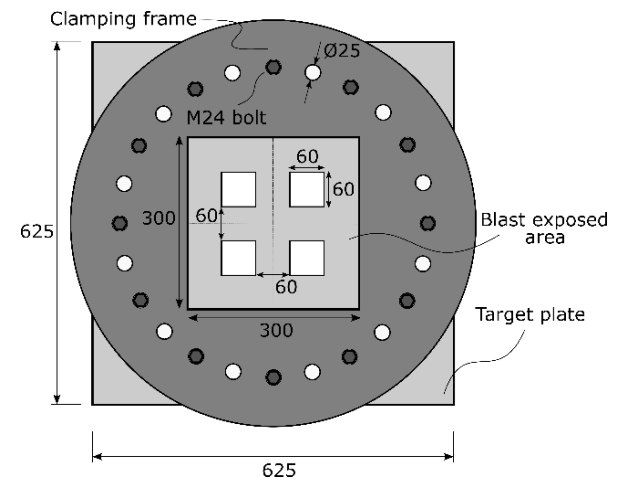

(b)

Fig. 2. The SIMLab Shock Tube Facility (SSTF) [9]: (a) a sketch of the entire facility and (b) the geometry of the clamping assembly and the blast-loaded target plates with pre-formed square holes.

\section{Numerical model}

All simulations in this study were performed in the explicit finite element code LS-DYNA. The material behaviour is described through a thermoviscoplastic constitutive model, which was implemented as a user-defined material model in LS-DYNA. The model is capable of describing large plastic strains, high strain rates and possible temperature softening, and is given by:

$$
\sigma_{e q}=\left(\sigma_{0}+R(p)\right)\left(1+\dot{p}^{*}\right)^{c}\left(1-T^{*^{m}}\right)
$$

where $\sigma_{e q}$ denotes the equivalent flow stress, given by a Hershey yield surface expressed in terms of the principal stresses $\sigma_{i}$ as:

$$
\sigma_{e q}=\left(\left|\sigma_{1}-\sigma_{2}\right|^{n}+\left|\sigma_{2}-\sigma_{3}\right|^{n}+\left|\sigma_{3}-\sigma_{1}\right|^{n}\right)^{\frac{1}{n}}
$$

where $n$ determines the shape of the yield surface. The von Mises yield surface is obtained by setting $n=2$, and Tresca by either $n=1$ or $n=\infty$. $R(p)$ describes the work hardening 
as a function of the equivalent plastic strain $p$, which is described by a three-term Voce hardening law on the form:

$$
R(p)=\sum_{k=1}^{3} Q_{k}\left(1-e^{-c_{k} * p}\right)
$$

The initial yield stress is given by $\sigma_{0}$, and $\left(Q_{k}, c_{k}\right)$ are hardening parameters. The exponents $c$ and $m$ are constants governing the rate and temperature dependency of the material. The dimensionless plastic strain rate is given by $\dot{p}^{*}=\dot{p} / \dot{p}_{0}$, where $\dot{p}_{0}$ is the reference strain rate used in the quasi-static tension tests. In this study, $n=6$ was applied for the yield surface and $m=1$ for the temperature softening part for both materials.

Finally, ductile fracture is accounted for by the energy-based fracture criterion proposed by Cockcroft-Latham [10]

$$
D=\frac{W}{W_{c}}=\frac{1}{W_{c}} \int_{0}^{p}\left\langle\sigma_{1}\right\rangle d p
$$

where $\sigma_{1}$ denotes the major principal stress, $W$ is the accumulated damage, and $W_{c}$ is the critical damage level leading to fracture in the model. $D$ then represents the normalized damage parameter, and element erosion is used to delete the element when $D=1$.

The critical damage $W_{c}$ was determined for each material and mesh by inverse modelling of the quasi-static tension tests to fracture The hardening parameters $\left(Q_{k}, c_{k}\right)$ were found by an optimalization of the numerical simulation of the tension tests. The strain rate sensitivity parameters were taken from Ref. [11]. All material parameters are given in Table 2.

Table 2. The material parameters applied for both materials in this study.

\begin{tabular}{|c|c|c|c|c|c|c|c|c|c|}
\hline Material & $\begin{array}{c}\sigma_{0} \\
{[\mathrm{MPa}]}\end{array}$ & $\begin{array}{c}Q_{1} \\
{[\mathrm{MPa}]}\end{array}$ & $\begin{array}{c}C_{1} \\
{[-]}\end{array}$ & $\begin{array}{c}Q_{2} \\
{[\mathrm{MPa}]}\end{array}$ & $\begin{array}{c}C_{2} \\
{[-]}\end{array}$ & $\begin{array}{c}Q_{3} \\
{[\mathrm{MPa}]}\end{array}$ & $\begin{array}{c}C_{3} \\
{[-]}\end{array}$ & $\begin{array}{c}c \\
{[-]}\end{array}$ & $\begin{array}{c}W_{c} \\
{[\mathrm{MPa}]}\end{array}$ \\
\hline Docol 600DL & 303.3 & 68.3 & 849.1 & 272.4 & 30.2 & 825.9 & 1.2 & 0.01 & 865.0 \\
\hline Docol1400M-1 & 1072.9 & 211.7 & 1504.8 & 195.6 & 231.7 & 76.1 & 79.4 & 0.004 & 425.0 \\
\hline Docol1400M-2 & 1150.0 & 309.5 & 1061.0 & 113.0 & 131.7 & 9061.0 & 0.09 & 0.004 & 452.0 \\
\hline
\end{tabular}

\section{Results}

The global response of thin steel plates exposed to blast loading has been evaluated thoroughly in Refs. $[7,8,12]$, and the focus in this study is directed more towards fracture modes and crack propagation. Further, a detailed numerical study on the Docol 600DL material was performed in Ref. [7]. The numerical simulations were therefore limited to the tests on the Docol 1400M material in this study.

Experimentally, the behaviour of the six blast tests yielded three distinct responses of the target plates: plastic deformations without any signs of fracture, plastic deformations and crack initiation, and complete fracture. In Refs. [7,8], it was observed that the Docol 600DL material experienced plastic deformations and crack initiation at the extremities of the holes for the 15 bar and 25 bar firing pressures, while the highest firing pressure of 35 bar resulted in complete fracture of the target plate with cracks along the diagonals. In this study, the Docol $1400 \mathrm{M}$ plates resulted in small plastic deformations without any visual crack initiation for the 15 bar firing pressure, and complete fracture when the firing pressure was increased to 25 bar and 35 bar. The reduced ductility in Docol $1400 \mathrm{M}$ seems to reduce the plates capacity to arrest the crack propagation, while the Docol 600DL plates experienced only crack initiation at the lowest and intermediate firing pressures. Thus, an increase in the 
material strength resulted in a reduced capacity against ductile fracture during blast loading. The final deformation or fracture mode of all six blast experiments are shown in Fig. 3.
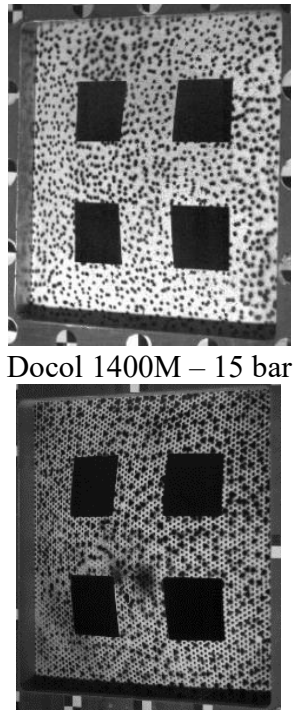

Docol 600DL - 15 bar
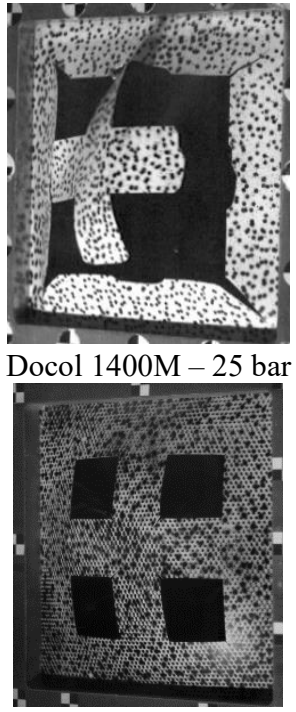

Docol 600DL - 25 bar
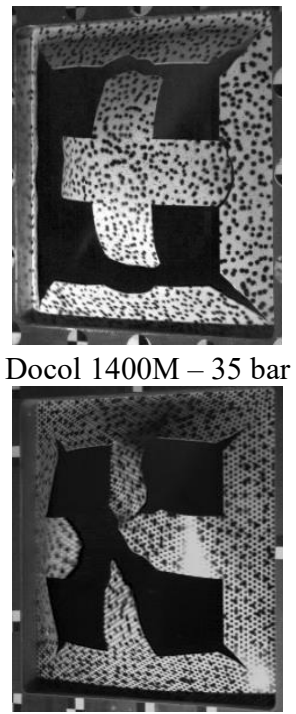

Docol 600DL - 35 bar

Fig. 3. Experimental results for all six experiments. Both the material and firing pressure are indicated in the sub-caption of each image.

All target plates were discretized using four solid elements across the thickness, corresponding to an element size of $0.2 \mathrm{~mm}$. The full clamping assembly, shown in Fig. $2 \mathrm{~b}$ was modelled, and quarter symmetry was utilized. The experimental results indicate that the 15 bar firing pressure for the Docol $1400 \mathrm{M}$ material is very close to the limit for crack initiation and ductile fracture. Therefore, it was further decided to run this simulation using material calibrations for two of the tension tests in the 0 -direction, with different levels of flow stress and elongation to fracture (see Fig. 1 and Table 2).

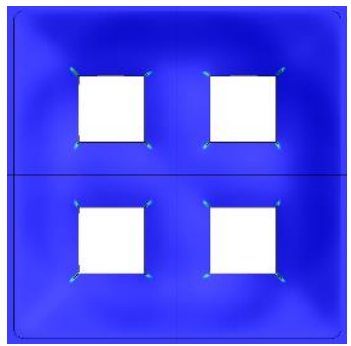

(a)

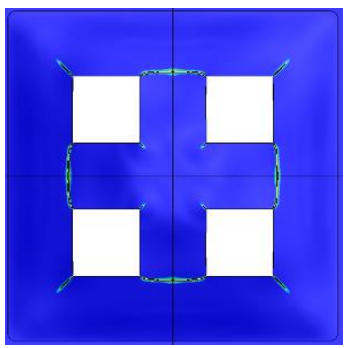

(b)

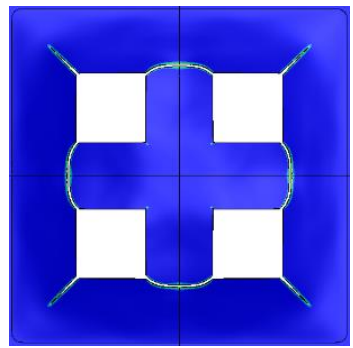

(c)

Fig. 4. Numerical results for the blast experiments on the Docol $1400 \mathrm{M}$ material for a firing pressure of 15 bar in (a) and (b), and 25 bar in (c). In (a) the fitted parameters for the Docol 1400M-2 was applied, while the material parameters for Docol 1400M-1 were used in (b).

As seen in Fig. 4c, the numerical model was capable of describing the fracture mode observed in the experiments for the 25 bar firing pressure. It is noted that an identical failure mode was also obtained for the simulation of the 35 bar firing pressure. For the lowest firing pressure of 15 bar, two different results were found based on the choice of stress-strain curves for the calibration of material parameters. As seen in Fig. 4 (a) and (b), the material parameters corresponding to the tension test in the 0 -direction with the largest elongation to 
fracture (Docol 1400M-2) resulted in small arrested cracks, while the material parameters corresponding to the test in the 0 -direction with the smallest elongation to fracture (Docol $1400 \mathrm{M}-1)$ resulted in complete fracture at the same firing pressure. Hence, a strong sensitivity to the choice of experimental stress-strain curve for material calibration was found. This effect was however not investigated any further in this study and is suggested as further work.

\section{Concluding remarks}

The present study investigates the influence of material properties on the blast performance of thin steel plates with pre-cut defects. Such effects were studied by combining experimental and numerical results. The main conclusions from the study are as follows.

- The steel plates with the lowest strength and the highest ductility (Docol 600DL) showed an increased capacity against fracture during blast loading compared to the steel plates made of the stronger and less ductile Docol 1400M material.

- The steel materials under consideration resulted in distinct differences in fracture mode when exposed to the same blast intensity. This behaviour was also captured by the numerical simulations.

- The Docol 1400M material showed a stronger sensitivity to the choice of material curve used in the calibration of the material parameters than the Docol 600DL material.

- It is emphasized that this work is ongoing research and that the presented results should be used with caution. More detailed studies are planned as further work.

\section{References}

1. W. Wilkinson, D. Cormie, M. Arkinstall. Proc. Inst. Civ. Eng. Eng. Comput. Mech. 166 (2013) 132-139.

2. L. Li, Q.-C. Zhang, R. Zhang, X. Wang, Z.-Y. Zhao, S.-Y. He, B. Han, T. J. Lu. Int. J. Impact Eng. 134 (2019) 103382.

3. W. Li, P. Wang, G.-P. Feng, Y.-G. Lu, J.-Z. Yue, H.-M. Li. Def. Technol. In Press (2020).

4. Y. Wei, Y. Li, L. Zhu, Y. Liu, X. Lei, G. Wang, Y. Wu, Z. Mi, J. Liu, H. Wang, H. Gao. Nat. Commun. 5 (2014) 3580.

5. SSAB Swedish Steel Ltd. https://www.ssab.com/products/brands/docol/docol-productoverview [accessed 22.03.21].

6. C. O. Paulsen. Experimental characterization of two-phase steels. Ph.D. thesis, NTNU (2019).

7. V. Aune, G. Valsamos, F. Casadei, M. Langseth, T. Børvik. Int. J. Impact Eng. 108 (2017) 27-46.

8. B. S. Elveli, M. Iddberg, T. Børvik, V. Aune. To be submitted (2021).

9. V. Aune, E. Fagerholt, M. Langseth, T. Børvik. Int. J. Protective Struct. 7 (2016) 340366.

10. M. G. Cockcroft, D. J. Latham. J. Inst. Met. 96 (1968) 33-39.

11. G. Gruben, M. Langseth, E. Fagerholt, O. Hopperstad. Int. J. Impact Eng. 88 (2016) 153171.

12. H. Granum, V. Aune, T. Børvik, O.S. Hopperstad. Int. J. Impact Eng. 132 (2019) 103306. 